\title{
Assessment of Nurses's Work Stress at EL- Minia University Hospitals
}

\author{
Heba. D. Ali , Fadia. A. El-Hameed, Sahar. A. Abood, and Nahed. S. Abo EL-Magd \\ Nursing Administration, Faculty of Nursing, El-Minia University, Egypt \\ Public health and preventive Medicine, Faculty of Medicine, El-Minia University, Egypt \\ Nursing Administration, Faculty of Nursing, El-Minia University, Egypt \\ Nursing Administration, Faculty of Nursing, Assiut University, Egypt
}

\begin{abstract}
:
Nursing is a stressful career; occupational stress is a common occurrence in health professions. Job stressors and low job control have been shown to be risk factors for patient safety and lead to poor job performance including reduced quality of nursing care. This study aimed to identify causes, level, and frequency of work stress on nurses at ELMinia University Hospitals. Methodology: Research design: The study was developed within descriptive approach. Setting: The study was carried out at EL-Minia and Suzan Mubarak University Hospitals in El-Minia Governorate. Subjects: All nurses (158) who worked in all general and critical departments at El-Minia University Hospital $(n=115)$ and Suzan Mubarak University Hospital $(n=43)$ in the period from 1 July 2011 to 30 November 2011. Tools of study: The study tools consisted of two tools; they include 1- Demographic data sheet 2- stress scale. Results: Less than two thirds of the studied sample had moderate level of stress; more than two thirds of the studied sample had severe level of the stress. Conclusions: There were statistically significant relation between level of education and stress level in the two hospitals. While there were no statistically significant differences between other demographic characteristics and stress levels in the two hospitals, and the most common causes of stress with the nurses who worked at El-Minia and Suzan Mubarak University Hospitals are considered death, dying, work load, and uncertainty concerning treatment respectively. Recommendations: Organizing stress management program that focuses on different categories of nurses at all hierarchical levels
\end{abstract}

Key Words: Stress ,Nurse,Work.

\section{Introduction}

Occupational stress occurrence is a common in health professions throughout the world. The National Health Services (NHS) in the United Kingdom (UK) and in Australia reported that occupational stress occurred among health professionals at higher levels than in any other comparable profession. This higher level of stress in health service has been attributed to the nature of the work of health professionals in which nurses, physicians and hospital administrators are involved in providing help to people experiencing life crises (Tyson \& Pongruengphant, 2004).

Individuals had been exposed to stress within all work environments today has been estimated to cost economy upwards of $\$ 300$ billion in sick time, long-term disability, and excessive job turnover. When an individual exposed to severe or chronic work stress in the work environment, a formal diagnosis of adjustment disorder may be made (Leiter \& Maslach, 2005, Devente et al., 2006). Chou et al., (2003) concluded that improved nursing staff competence and work satisfaction and decreased work stress would be reflected in improved quality of care ratings.

Lack of productivity due to occupational stress and its related effects, including staff conflicts, recruitment and retention problems, burnout, absenteeism, rapid turn-over, and lack of job satisfaction, has been reported to cause significant monetary costs. The [NHS] reported that organizations spend as much as $\$ 75$ billion a year on stress-related outcomes including physical injuries at work and absenteeism; while the World Health Organization estimates the cost of stress and stressrelated problems to organizations to be in excess of $\$ 150$ billion annually (Alves, 2005).

Sveinsdottir et al., (2006) clarified that job stressors (causes of stress) and low job control have been shown to be risk factors for patient safety and lead to poor job performance including reduced quality of nursing care. Hannan et al., (2001) concluded that work stressor experienced by health care staff have been found to adversely affect job performance and negatively impact the quality of care given to patients and long term care residents .

Understanding the stressful work environments of nurses is vital in addressing the nursing shortage. Transformation of the critical care work environment is essential in order to: retain and recruit nurses, improve job satisfaction, and improve patient outcomes (McCauley \& Irwin, 2006).

Job stress is an acknowledged phenomenon in the professional practice of nursing. The relevance of job stress for nursing is in its relationship to nurse 
burnout, workforce absenteeism, retention and turnover and patient outcomes (Laschinger \& Leiter, 2006).

In Egypt many studies have investigated stress such as (Mohamed et al., 2011) who concluded that nurses in the Children's University Hospital at Elshatby are confronted with a multitude of stressful clinical situations. The most intense and frequent stress was associated with death and dying, followed by uncertainty about treatment, conflict with other nurses, and workload. Also nurses are confronted with multiple stress factors which are: personal, interpersonal relationship, health care system, occupational and environment stress factors.

\section{The aims of the study:}

1. Identify causes of work stress from nurses point of view in both hospitals.

2. Identify level of work stress among nurses in both hospitals.

3. Identify frequency of work stress occurrences among nurses in both hospitals.

\section{Significance of the study:}

During clinical training of the nursing students in EL-Minia University Hospitals, the researcher observed that there are high job demands, lack of supportive work relationships, shortage of equipment and supplies, and work overload among nurses which may lead to inadequate nursing care activities. Also noticed that nurses are always stressed this make the researcher think to make the research.

\section{Subjects and Methods}

The methodology pursued in the conduction of the study will be described under four designs, namely technical, operational, administrative, and statistical designs.

Technical design: This design involves the research design, the setting, subject, and tools of data collection.

\section{Research design:}

The study was developed within a descriptive approach.

\section{Setting:}

The study was carried out in EL-Minia University Hospital and Suzan Mubarak University Hospital in El-Minia Governorate. EL-Minia University Hospital was built in 1988 to replace the old Ministry of Health Hospital, which has been used by the Faculty of Medicine as a teaching hospital since1987. All medical, surgical departments and intensive care units of this hospital were included in the study. The bed capacity of the hospital is six hundred ninety two beds and the total numbers of the nursing staff were classified as the following; three hundred ninety nine staff nurses, twenty seven head nurses, twenty two supervisors and two director. The hospital included eighteen inpatient units, fifteen outpatient units and sixteen units of special character.

Suzan Mubarak University Hospital was built in 2005 which has been used by the Faculty of Medicine as a teaching hospital. Obstetric, pediatric departments and intensive care units were included in the study. The bed capacity of the hospital is three hundred six beds and the total number of the nursing were classified as the following; ninety nine staff nurses, ten head nurses, five supervisors and two director.

\section{Subject:}

The sample consists of all nurses) both staff and head nurse) who are working in all general inpatient, and critical care units at EL-Minia University Hospital $(\mathrm{n}=115)$ and Suzan Mubarak University Hospital $(\mathrm{n}=$ 43) with total number $(n=158)$. Their age ranged between19 to 58 years old. Their nursing qualifications were diploma, technical institute, and baccalaureate degree. They are classified as the following: 


\begin{tabular}{|c|c|c|c|}
\hline Hospital name & Unit & Type & Number of nurses \\
\hline \multirow{14}{*}{$\begin{array}{c}\text { EL-Minia } \\
\text { university hospital }\end{array}$} & Surgical department. & \multirow{11}{*}{$\begin{array}{c}\text { General } \\
\text { area }\end{array}$} & 13 \\
\hline & Orthopedic department. & & 4 \\
\hline & Urology department & & 5 \\
\hline & ENT department & & 4 \\
\hline & Ophthalmology department. & & 5 \\
\hline & Special medical departments & & 5 \\
\hline & Tropical department. & & 5 \\
\hline & General medical department. & & 11 \\
\hline & Recovery department. & & 8 \\
\hline & Health insurance department (1) & & 5 \\
\hline & Health insurance department (2) & & 8 \\
\hline & Medical intensive care unit & \multirow{3}{*}{$\begin{array}{c}\text { Critical } \\
\text { area }\end{array}$} & 9 \\
\hline & Coronary care unit (CCU) & & 21 \\
\hline & Intensive care unit (ICU) & & 12 \\
\hline \multirow{4}{*}{$\begin{array}{l}\text { Suzan } \\
\text { Mubarak } \\
\text { University } \\
\text { Hospital }\end{array}$} & Obstetric department & \multirow{2}{*}{$\begin{array}{c}\text { General } \\
\text { area }\end{array}$} & 11 \\
\hline & Pediatric department & & 9 \\
\hline & Neonatal intensive care unit (NICU) & \multirow{2}{*}{$\begin{array}{c}\text { Critical } \\
\text { area }\end{array}$} & 14 \\
\hline & (Pediatric\& Obstetric) intensive care unit & & 9 \\
\hline \multicolumn{2}{|r|}{ Total } & & 158 \\
\hline
\end{tabular}

Tools of data collection:

Data for the present study were collected using the following tools. These were a demographic data, and stress scale.

\section{1) Demographic data:}

Demographic characteristics about study nurses working at EL-Minia and Suzan Mubarak University Hospitals. It includes (name, age, sex, marital status, qualification, years of experience, department, and kind of shifts nurses taken).

\section{2) Stress scale:}

This tool was developed by (Cassem and Hakett 1981) measures sources and frequency of workplace stress, and it was translated by the researcher. It included 34 questions about stress; it is divided into the following sequence 7 factors:

- $\quad$ Death and dying (7items)

- Conflict with Physician (5 items)

- Inadequate preparation (3 items)

- Lack of support (3 items)

- Conflict with other nurses (5 items)

- Workload (6 items)

- Uncertainty concerning treatment (5 items).

Scoring system: Responses with a four point dimension from 1 to 4 [never stressful= (1), occasionally stressful $=(2)$, frequently stressful $=(3)$, extremely stressful $=(4)]$. So the scoring system ranges from 34 to 136 and divided as follows; If the score (34) the level of stress will be considered no, and if the score ranges from (35- 68) the level of stress will be considered mild, if ranges from (69102) the level of stress will be considered moderate, and if ranges from (103-136) the level of stress will be considered severe.

\section{i. Administrative design:}

An official permission was taken from Dean of Faculty of Nursing-El-Minia University and from the managers of (EL-Minia and Suzan Mubarak University Hospitals).

\section{Ethical consideration:}

Oral agreement was taken from the participating nurses in the Hospitals (EL-Minia and Suzan Mubarak University Hospitals). Confidentiality of obtained data was assured, the purpose, nature, and the aim of the study was explained to all participating nurses before starting data collection.

\section{iii. Operational design:-}

Preparation phase: This phase started from March 2011 to June 2011 and included the following:

1 - Reviewing the available literature concerning the topic of the study.

2- Preparing and translating of the study's tool.

This phase took four months from March to June 2011 the researcher spent this time in reviewing the available literature concerning the topic of the study and Arabic translation of the stress tool.

Validity: The stress tool were translated and checked for its content validity through a pilot study by 11 jury from teaching staff of Faculty of Nursing- 
Assuit University and teaching staff of Faculty of Nursing-El-Minia University (two professors from Community Department, one professor from Obstetric Department, five professors from Administration Department and three professors from Geriatric Department).

Pilot study: A pilot study was carried out on group of sixteen nurses from the hospitals before embarking on the field work to find out the difficulties present in questions in order to modify or clarify them, or to omit or add certain questions, and to estimate time needed to fill the forms. Also pilot study helped to determine the organizational and administrative procedures needed for the study, and detect difficulties that may arise and how to deal with them. Modifications and clarifications were done according to the pilot study result. Minor modifications were done, and the final form was developed. The results obtained from the pilot study were included in the main sample. The reliability Alpha co-efficient of the total stress tool was $(\alpha=0.79)$.
Filed work: The present study carried out within five months started from 1 July 2011 to 30 November 2011.

The stress scale: The researcher met each nurse to explain the purpose of the study and to ask for participation. After obtaining verbal consent, the stress scale handles to participated nurses to be filled. The data collection was done through structured questionnaire with participants to fill the forms. This took about twenty minutes for each participant interview.

iv. Statistical design: The collected data were thoroughly entered and then, tabulated, analyzed, and interpreted by using frequency distribution percentages, chi-square test and Fisher' exact test whenever, applicable. All data were analyzed by using the Statistical Package for Social Sciences (SPSS-16) software and $\mathrm{P}<0.05$ was used as the definition of statistical significance.

\section{Results:}

Table (1) Frequency distribution of personal characteristics of nurses in the study sample in El-Minia and Suzan Mubarak University Hospitals, 2011.

\begin{tabular}{|c|c|c|c|c|c|c|c|c|c|c|c|c|}
\hline \multirow{3}{*}{$\begin{array}{l}\text { Demographic } \\
\text { characteristics }\end{array}$} & \multicolumn{6}{|c|}{$\begin{array}{l}\text { El- Minia University Hospital } \\
\text { (No.=115) }\end{array}$} & \multicolumn{6}{|c|}{$\begin{array}{c}\text { Suzan Mubarak University Hospital } \\
(\text { No. }=43)\end{array}$} \\
\hline & \multicolumn{2}{|c|}{$\begin{array}{l}\text { General } \\
\text { (73) }\end{array}$} & \multicolumn{2}{|c|}{$\begin{array}{c}\text { Critical } \\
(42)\end{array}$} & \multicolumn{2}{|c|}{$\begin{array}{l}\text { Total } \\
(115)\end{array}$} & \multicolumn{2}{|c|}{$\begin{array}{l}\text { General } \\
(\mathbf{2 0})\end{array}$} & \multicolumn{2}{|c|}{$\begin{array}{c}\text { Critical } \\
(23)\end{array}$} & \multicolumn{2}{|c|}{$\begin{array}{c}\text { Total } \\
(43)\end{array}$} \\
\hline & No & $\%$ & No & $\%$ & No & $\%$ & No & $\%$ & No & $\%$ & No & $\%$ \\
\hline 1. Age & & & & & & & & & & & & \\
\hline $\begin{array}{ll} & <20 \\
\text { - } & 20-29 \\
\text { - } & 30-39 \\
\text { - } & 40-49\end{array}$ & $\begin{array}{c}2 \\
37 \\
25 \\
9\end{array}$ & $\begin{array}{c}2.7 \\
50.7 \\
34.2 \\
12.4\end{array}$ & $\begin{array}{c}0 \\
39 \\
2 \\
1\end{array}$ & $\begin{array}{c}0.0 \\
92.8 \\
4.8 \\
2.4\end{array}$ & $\begin{array}{c}2 \\
76 \\
27 \\
10\end{array}$ & \begin{tabular}{c|}
1.7 \\
66.1 \\
23.5 \\
8.7 \\
\end{tabular} & $\begin{array}{c}0 \\
18 \\
2 \\
0\end{array}$ & $\begin{array}{c}0.0 \\
90.0 \\
10.0 \\
0.0\end{array}$ & $\begin{array}{c}0 \\
20 \\
3 \\
0\end{array}$ & $\begin{array}{c}0.0 \\
87.0 \\
130 \\
0.0\end{array}$ & $\begin{array}{c}0 \\
38 \\
5 \\
0\end{array}$ & $\begin{array}{c}0.0 \\
\mathbf{8 8 . 4} \\
11.6 \\
0.0\end{array}$ \\
\hline 2. Sex & & & & & & & & & & & & \\
\hline $\begin{array}{ll} & \text { Male } \\
\text { - } & \text { Female } \\
\end{array}$ & $\begin{array}{l}29 \\
44 \\
\end{array}$ & $\begin{array}{l}39.7 \\
60.3 \\
\end{array}$ & $\begin{array}{c}7 \\
35 \\
\end{array}$ & $\begin{array}{l}16.7 \\
83.3 \\
\end{array}$ & $\begin{array}{l}36 \\
79 \\
\end{array}$ & $\begin{array}{l}31.3 \\
\mathbf{6 8 . 7} \\
\end{array}$ & $\begin{array}{c}0 \\
20 \\
\end{array}$ & $\begin{array}{l}0.0 \\
100 \\
\end{array}$ & $\begin{array}{c}0 \\
23 \\
\end{array}$ & $\begin{array}{l}0.0 \\
100 \\
\end{array}$ & $\begin{array}{c}0 \\
43 \\
\end{array}$ & $\begin{array}{l}0.0 \\
\mathbf{1 0 0} \\
\end{array}$ \\
\hline 3.Marital status & & & & & & & & & & & & \\
\hline $\begin{array}{ll}- & \text { Single } \\
- & \text { Married } \\
\end{array}$ & $\begin{array}{l}17 \\
56\end{array}$ & $\begin{array}{l}23.3 \\
76.7\end{array}$ & $\begin{array}{l}28 \\
14 \\
\end{array}$ & $\begin{array}{l}66.7 \\
33.3\end{array}$ & $\begin{array}{l}45 \\
70\end{array}$ & $\begin{array}{l}39.1 \\
\mathbf{6 0 . 9}\end{array}$ & $\begin{array}{c}8 \\
12 \\
\end{array}$ & $\begin{array}{l}40.0 \\
60.0\end{array}$ & $\begin{array}{l}11 \\
12 \\
\end{array}$ & $\begin{array}{l}47.8 \\
52.2\end{array}$ & $\begin{array}{l}19 \\
24\end{array}$ & $\begin{array}{l}44.2 \\
\mathbf{5 5 . 8}\end{array}$ \\
\hline 4.Qualifications & & & & & & & & & & & & \\
\hline $\begin{array}{l}\text { - Diploma } \\
\text { - Institute } \\
\text { - Baccalaureate }\end{array}$ & $\begin{array}{c}59 \\
14 \\
0\end{array}$ & $\begin{array}{c}80.8 \\
19.2 \\
0.0\end{array}$ & $\begin{array}{l}13 \\
13 \\
16\end{array}$ & $\begin{array}{l}31.0 \\
31.0 \\
38.0\end{array}$ & $\begin{array}{l}72 \\
27 \\
16\end{array}$ & $\begin{array}{l}\mathbf{6 2 . 6} \\
23.5 \\
13.9\end{array}$ & $\begin{array}{c}17 \\
3 \\
0\end{array}$ & $\begin{array}{c}85.0 \\
15.0 \\
0.0\end{array}$ & $\begin{array}{l}9 \\
6 \\
8\end{array}$ & $\begin{array}{l}39.1 \\
26.1 \\
34.8\end{array}$ & $\begin{array}{c}26 \\
9 \\
8\end{array}$ & $\begin{array}{l}\mathbf{6 0 . 5} \\
20.9 \\
18.6\end{array}$ \\
\hline $\begin{array}{l}\text { 6.Years of } \\
\text { experience }\end{array}$ & & & & & & & & & & & & \\
\hline $\begin{array}{ll}\cdot & <10 \\
\text { - } & 10-20 \\
\text { - } & >20\end{array}$ & $\begin{array}{c}39 \\
26 \\
8\end{array}$ & $\begin{array}{c}54.2 \\
36.1 \\
9.7\end{array}$ & $\begin{array}{c}40 \\
2 \\
0\end{array}$ & $\begin{array}{c}95.2 \\
4.8 \\
0.0\end{array}$ & $\begin{array}{c}79 \\
28 \\
7\end{array}$ & $\begin{array}{c}\mathbf{6 9 . 3} \\
24.6 \\
6.1\end{array}$ & $\begin{array}{c}16 \\
4 \\
0\end{array}$ & $\begin{array}{c}80.0 \\
20.0 \\
0.0\end{array}$ & $\begin{array}{c}20 \\
3 \\
0\end{array}$ & $\begin{array}{c}87.0 \\
13.0 \\
0.0\end{array}$ & $\begin{array}{c}36 \\
7 \\
0\end{array}$ & $\begin{array}{c}\mathbf{8 3 . 7} \\
16.3 \\
0.0\end{array}$ \\
\hline
\end{tabular}


Table (2) Frequency of causes of stress among nurses in El-Minia and Suzan Mubarak University Hospitals, 2011.

\begin{tabular}{|c|c|c|c|c|c|}
\hline \multirow{2}{*}{\multicolumn{2}{|c|}{ frequency of causes of stress }} & \multicolumn{2}{|c|}{ El-Minia } & \multicolumn{2}{|c|}{ Suzan } \\
\hline & & $\mathrm{No}=115$ & $\%$ & $\mathrm{No}=43$ & $\%$ \\
\hline \multirow{4}{*}{ Death and dying } & Never & 0 & 0.0 & 0 & 0.0 \\
\hline & Occasionally & 4 & 3.5 & 0 & 0.0 \\
\hline & Frequently & 38 & 33.0 & 15 & 34.9 \\
\hline & Extremely & 73 & 63.5 & 28 & 65.1 \\
\hline \multirow{4}{*}{$\begin{array}{c}\text { Conflict with } \\
\text { physicians }\end{array}$} & Never & 0 & 0.0 & 0 & 0.0 \\
\hline & Occasionally & 19 & 16.5 & 5 & 11.6 \\
\hline & Frequently & 59 & 51.3 & 23 & 53.5 \\
\hline & Extremely & 37 & 32.2 & 15 & 34.9 \\
\hline \multirow{4}{*}{ Lack of support } & Never & 7 & 6.0 & 3 & 7.0 \\
\hline & Occasionally & 34 & 29.5 & 11 & 25.5 \\
\hline & Frequently & 50 & 43.6 & 27 & 62.8 \\
\hline & Extremely & 24 & 20.9 & 2 & 4.7 \\
\hline \multirow{4}{*}{$\begin{array}{l}\text { Conflict with } \\
\text { other nurses }\end{array}$} & Never & 1 & 0.9 & 0 & 0.0 \\
\hline & Occasionally & 25 & 21.7 & 5 & 11.6 \\
\hline & Frequently & 58 & 50.4 & 19 & 44.2 \\
\hline & Extremely & 31 & 27.0 & 19 & 44.2 \\
\hline \multirow{4}{*}{ Work load } & Never & 0 & 0.0 & 0 & 0.0 \\
\hline & Occasionally & 5 & 4.3 & 2 & 4.7 \\
\hline & Frequently & 43 & 37.4 & 15 & 34.8 \\
\hline & Extremely & 67 & 58.3 & 26 & 60.5 \\
\hline \multirow{4}{*}{$\begin{array}{l}\text { Uncertainty } \\
\text { concerning } \\
\text { treatment }\end{array}$} & Never & 0 & 0.0 & 0 & 0.0 \\
\hline & Occasionally & 11 & 9.6 & 3 & 7.0 \\
\hline & Frequently & 48 & 41.7 & 20 & 46.5 \\
\hline & Extremely & 56 & 48.7 & 20 & 46.5 \\
\hline
\end{tabular}


Table (3) Relation between stress level of nurses at El-Minia and Suzan Mubarak University Hospitals and their working areas (general and critical) by Hospitals name, 2011.

\begin{tabular}{|c|c|c|c|c|c|c|c|c|c|c|c|c|}
\hline \multirow[t]{3}{*}{ Stress level } & \multicolumn{6}{|c|}{ El- Minia University Hospital } & \multicolumn{6}{|c|}{ Suzan Mubarak University Hospital } \\
\hline & \multicolumn{2}{|c|}{ General } & \multicolumn{2}{|c|}{ Critical } & \multicolumn{2}{|c|}{ Total } & \multicolumn{2}{|c|}{ General } & \multicolumn{2}{|c|}{ Critical } & \multicolumn{2}{|c|}{ Total } \\
\hline & No & $\%$ & No & $\%$ & No & $\%$ & No & $\%$ & No & $\%$ & No & $\%$ \\
\hline No & 0 & 0.0 & 0 & 0.0 & 0 & $\mathbf{0 . 0}$ & 0 & 0.0 & 0 & 0.0 & 0 & $\mathbf{0 . 0}$ \\
\hline Mild & 3 & 4.1 & 1 & 2.4 & 4 & 3.5 & 0 & $\mathbf{0 . 0}$ & 0 & $\mathbf{0 . 0}$ & 0 & 0.0 \\
\hline Moderate & 44 & 60.3 & 28 & 66.6 & 72 & 62.6 & 10 & 50.0 & 17 & 73.9 & 27 & 62.8 \\
\hline Sever & 26 & 35.6 & 13 & 31.0 & 39 & 33.9 & 10 & 50.0 & 6 & 26.1 & 16 & 37.2 \\
\hline Total & 73 & 100 & 42 & 100 & 115 & 100 & 20 & 100 & 23 & 100 & 43 & 100 \\
\hline Fisher exact & \multicolumn{6}{|c|}{0.574} & \multicolumn{6}{|c|}{2.618} \\
\hline P value & \multicolumn{6}{|c|}{$0.750 \mathrm{NS}$} & \multicolumn{6}{|c|}{$0.106 \mathrm{NS}$} \\
\hline
\end{tabular}

$\mathrm{NS}=$ Not significant

Table (4) Relation between stress level and age of nurses in El-Minia and Suzan Mubarak University Hospitals, 2011.

\begin{tabular}{|c|c|c|c|c|c|c|c|c|c|c|c|c|}
\hline \multirow{3}{*}{$\begin{array}{l}\text { Age } \\
\text { group }\end{array}$} & \multicolumn{8}{|c|}{ Stress level } & & & \multirow{2}{*}{$\begin{array}{c}\text { Fisher } \\
\text { exact }\end{array}$} & \multirow{2}{*}{$\begin{array}{c}P \\
\text { value }\end{array}$} \\
\hline & \multicolumn{2}{|c|}{ No } & \multicolumn{2}{|c|}{ Mild } & \multicolumn{2}{|c|}{ Moderate } & \multicolumn{2}{|c|}{ Sever } & \multicolumn{2}{|c|}{ Total } & & \\
\hline & $\overline{\mathrm{No}}$ & $\%$ & $\overline{\text { No }}$ & $\%$ & $\overline{\mathrm{No}}$ & $\%$ & No & $\%$ & No & $\%$ & & \\
\hline$<20$ & 0 & 0.0 & 0 & 0.0 & 1 & 50.0 & 1 & $\overline{50.0}$ & 2 & 100 & & \\
\hline $20-29$ & 0 & 0.0 & 4 & 3.5 & 66 & 57.9 & 44 & 38.6 & 114 & 100 & 5202 & 0.496 \\
\hline 30-39 & 0 & 0.0 & 0 & 0.0 & 24 & 75.0 & 8 & 25.0 & 32 & 100 & 5.383 & NS \\
\hline 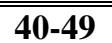 & 0 & 0.0 & 0 & 0.0 & 8 & 80.0 & 2 & 20.0 & 10 & 100 & & \\
\hline $\begin{array}{l}\text { Total } \\
\end{array}$ & 0 & 0.0 & 4 & 2.5 & 99 & 62.7 & 55 & 34.8 & 158 & 100 & & \\
\hline
\end{tabular}

Table (5) Relation between stress level and sex of nurses in both El-Minia and Suzan Mubarak University Hospitals, 2011.

\begin{tabular}{|c|c|c|c|c|c|c|c|c|c|c|c|c|}
\hline \multirow{3}{*}{ Sex } & \multicolumn{8}{|c|}{ Stress level } & \multirow{2}{*}{\multicolumn{2}{|c|}{ Total }} & \multirow{2}{*}{$\begin{array}{c}\text { Fisher } \\
\text { exact }\end{array}$} & \multirow{2}{*}{$\begin{array}{c}\mathbf{P} \\
\text { value }\end{array}$} \\
\hline & \multicolumn{2}{|c|}{ No } & \multicolumn{2}{|c|}{ Mild } & \multicolumn{2}{|c|}{ Moderate } & \multicolumn{2}{|c|}{ Sever } & & & & \\
\hline & No & $\%$ & No & $\%$ & No & $\%$ & No & $\%$ & No & $\%$ & \multirow{4}{*}{3.267} & \multirow{4}{*}{$\begin{array}{c}0.195 \\
\text { NS }\end{array}$} \\
\hline Male & 0 & 0.0 & 1 & 2.8 & 27 & 75.0 & 8 & 22.2 & 36 & 100 & & \\
\hline Female & 0 & $\mathbf{0 . 0}$ & 3 & 2.5 & 72 & 59.0 & 47 & 38.5 & 122 & 100 & & \\
\hline Total & 0 & 0.0 & 4 & 2.5 & 99 & 62.7 & 55 & 34.8 & 158 & 100 & & \\
\hline
\end{tabular}

Table (6) Relation between stress level of nurses in both El-Minia and Suzan Mubarak University Hospitals and their marital status, 2011.

\begin{tabular}{|c|c|c|c|c|c|c|c|c|c|c|c|c|}
\hline \multirow{3}{*}{$\begin{array}{l}\text { Marital } \\
\text { status }\end{array}$} & \multicolumn{8}{|c|}{ Stress level } & \multirow{2}{*}{\multicolumn{2}{|c|}{ Total }} & \multirow{2}{*}{$\begin{array}{c}\text { Fisher } \\
\text { exact }\end{array}$} & \multirow{2}{*}{$\begin{array}{c}P \\
\text { value }\end{array}$} \\
\hline & \multicolumn{2}{|c|}{ No } & \multicolumn{2}{|c|}{ Mild } & \multicolumn{2}{|c|}{ Moderate } & \multicolumn{2}{|c|}{ Sever } & & & & \\
\hline & No & $\%$ & No & $\%$ & No & $\%$ & No & $\%$ & No & $\%$ & \multirow{4}{*}{5.217} & \multirow{4}{*}{$\begin{array}{c}0.074 \\
\text { NS }\end{array}$} \\
\hline Single & 0 & 0.0 & 3 & 4.7 & 34 & 53.1 & 27 & 42.2 & 64 & 100 & & \\
\hline Married & 0 & 0.0 & 1 & 1.1 & 65 & 69.1 & 28 & 29.8 & 94 & 100 & & \\
\hline Total & 0 & 0.0 & 4 & 2.5 & 99 & 62.7 & 55 & 34.8 & 158 & 100 & & \\
\hline
\end{tabular}


Table (7) Relation between stress level of nurses in both El-Minia and Suzan Mubarak University Hospitals and their qualifications, 2011.

\begin{tabular}{|c|c|c|c|c|c|c|c|c|c|c|c|c|}
\hline \multirow{3}{*}{ Qualifications } & \multicolumn{8}{|c|}{ Stress level } & \multirow{2}{*}{\multicolumn{2}{|c|}{ Total }} & \multirow{3}{*}{$\begin{array}{c}\text { Fisher } \\
\text { exact }\end{array}$} & \multirow{3}{*}{$\begin{array}{c}\mathbf{P} \\
\text { value }\end{array}$} \\
\hline & \multicolumn{2}{|c|}{ No } & \multicolumn{2}{|c|}{ Mild } & \multicolumn{2}{|c|}{ Moderate } & \multicolumn{2}{|c|}{ Sever } & & & & \\
\hline & No & $\%$ & No & $\%$ & No & $\%$ & No & $\%$ & No & $\%$ & & \\
\hline Diploma & 0 & 0.0 & 0 & 0.0 & 62 & 63.3 & 36 & 36.7 & 98 & 100 & \multirow{4}{*}{15.065} & \multirow{4}{*}{$\begin{array}{c}0.005 * \\
*\end{array}$} \\
\hline Institute & 0 & 0.0 & 4 & 11.1 & 23 & 63.9 & 9 & 25.0 & 36 & 100 & & \\
\hline Baccalaureate & 0 & 0.0 & 0 & 0.0 & 14 & 58.3 & 10 & 41.7 & 24 & 100 & & \\
\hline Total & 0 & 0.0 & 4 & 2.5 & 99 & 62.7 & 55 & 34.8 & 158 & 100 & & \\
\hline
\end{tabular}

(**) highly statistically significant at $<0.001$

Table (8) Relation between stress level of nurses in both El-Minia and Suzan Mubarak University Hospitals and their years of experiences, 2011.

\begin{tabular}{|c|c|c|c|c|c|c|c|c|c|c|c|c|}
\hline \multirow{3}{*}{$\begin{array}{c}\text { Years of } \\
\text { experien } \\
\text { ce }\end{array}$} & \multicolumn{8}{|c|}{ Stress level } & & & \multirow{3}{*}{$\begin{array}{l}\text { Fisher } \\
\text { exact }\end{array}$} & \multirow{3}{*}{$\begin{array}{c}\mathbf{P} \\
\text { value }\end{array}$} \\
\hline & \multicolumn{2}{|c|}{ No } & \multicolumn{2}{|c|}{ Mild } & \multicolumn{2}{|c|}{ Moderate } & \multicolumn{2}{|c|}{ Sever } & \multicolumn{2}{|c|}{ Total } & & \\
\hline & No & $\%$ & No & $\%$ & No & $\%$ & No & $\%$ & No & $\%$ & & \\
\hline$<10$ & 0 & 0.0 & 4 & 3.5 & 69 & 60.0 & 42 & 36.5 & 115 & 100 & & \\
\hline $10-20$ & 0 & 0.0 & 0 & 0.0 & 25 & 68.6 & 11 & 31.4 & 35 & 100 & 2.158 & 0.707 \\
\hline$>20$ & 0 & 0.0 & 0 & 0.0 & 5 & 71.4 & 2 & 28.6 & 7 & 100 & & NS \\
\hline Total & 0 & 0.0 & 4 & 2.5 & 99 & 62.7 & 55 & 34.8 & 158 & 100 & & \\
\hline
\end{tabular}

Table (9) Relation between stress level of nurses in both El-Minia and Suzan Mubarak University Hospitals and their shifts, 2011.

\begin{tabular}{|c|c|c|c|c|c|c|c|c|c|c|c|c|}
\hline \multirow{3}{*}{ Shift } & \multicolumn{8}{|c|}{ Stress level } & \multirow{2}{*}{\multicolumn{2}{|c|}{ Total }} & \multirow{3}{*}{$\begin{array}{l}\text { Fisher } \\
\text { exact }\end{array}$} & \multirow{2}{*}{$\begin{array}{c}P \\
\text { value }\end{array}$} \\
\hline & \multicolumn{2}{|c|}{ No } & \multicolumn{2}{|c|}{ Mild } & \multicolumn{2}{|c|}{ Moderate } & \multicolumn{2}{|c|}{ Sever } & & & & \\
\hline & No & $\%$ & No & $\%$ & No & $\%$ & No & $\%$ & No & $\%$ & & \multirow{5}{*}{$\begin{array}{c}0.062 \\
\text { NS }\end{array}$} \\
\hline Morning & 0 & 0 & 0 & 0 & 41 & 62.1 & 25 & 37.9 & 66 & 100 & \multirow{4}{*}{8.952} & \\
\hline Evening & 0 & 0 & 1 & 1.6 & 40 & 64.5 & 21 & 33.9 & 62 & 100 & & \\
\hline Night & 0 & $\mathbf{0}$ & 3 & 10.0 & 18 & 60.0 & 9 & 30.0 & 30 & 100 & & \\
\hline Total & 0 & 0 & 4 & 2.5 & 99 & 62.7 & 55 & 34.8 & 158 & 100 & & \\
\hline
\end{tabular}


Table (1) Describes the personal and job characteristics of nurses in the study sample by department at El-Minia and Suzan Mubarak University Hospitals. As evident in the table, about two thirds $(66.1 \%)$ of the study sample at El-Minia University Hospital, aged between 20-29 years, more than two thirds $(68.7 \%)$ of them were females, more than half $(60.9 \%)$ were married, less than two thirds $(62.6 \%)$ of them graduated from diploma Nursing and more than two thirds (69.3\%) had less than 10 years of experience.

As regard to Suzan Mubarak University Hospital, the majority of study sample (88.4\%) aged between 20 29 years, all of nurses were females $(100 \%)$, more than half of them were married $(55.8 \%)$, less than two thirds $(60.5 \%)$ of them graduated from diploma Nursing and the majority of study sample $(83.7 \%)$ had less than 10 years of experience

Table (2) Describes the frequency causes of stress among nurses in El-Minia and Suzan Mubarak University Hospitals. As evident in the table, death \& dying, work load and uncertainty concerning treatment were considered extremely stressful by most of the nurses of each hospital $(63.5 \%, 58.3 \%$ and 48.7 of nurses at El-Minia University Hospital and $65.1 \%, 60.5 \%$ and $46.5 \%$ of nurses at Suzan Mubarak University Hospital

Table (3) Describes the stress level of nurses in general and critical area by Hospitals name. As evident in the table, regarding El-Minia University Hospital, only $4.1 \%$ of nurses who worked in the general area versus $2.4 \%$ of those worked in the critical area had mild stress. More than one third $(35.6 \%)$ of nurses who worked in general area versus less than one third $(31.0 \%)$ of those worked in critical area had severe stress. And these differences were not statistically significant ( $\mathrm{p}$ value $=0.750$ ).

As regarding Suzan Mubarak University Hospital, none $(0 \%)$ of nurses who worked in the general and critical area had mild stress. Half $(50 \%)$ of nurses who worked in general area versus more than one quarter $(26.1 \%)$ of those worked in the critical area had severe stress. And these differences were not statistically significant ( $\mathrm{p}$ value $=0.106$ ).

respectively).

Table (4) Displays the relation between the stress level of nurses in both El-Minia and Suzan Mubarak University Hospitals and their age group. As regard to sever stress, nurses whose age $<20$ had the highest percentages $(50 \%)$, and as age increases the percent of severe stress decrease. These differences were not statistically significant ( $\mathrm{p}$ value $=0.496$ ).
Table (5)Male and female regarding stress level are compared in table (5). As evident in the table, three quarters $(75.0 \%)$ of male nurses, versus more than half $(59 \%)$ of female nurses had moderate stress. Less than quarter $(22.2 \%)$ of male nurses, versus more than one third $(38.5 \%)$ of female nurses had severe stress. These differences were not statistically significant $\mathrm{p}$ value $=.195$ ).

Table (6)Single and married nurses are compared regarding stress level in table (6). As evident in the table, more than half $(53.1 \%)$ of single nurses, versus more than two thirds $(69.1 \%)$ of married nurses had moderate stress. Less than half $(42.2 \%)$ of single nurses, versus less than one third $(29.8 \%)$ of married nurses had severe stress. These differences were not statistically significant $(\mathrm{p}$ value $=0.074)$.

Table (7) Describes the relation between level of education of nurses in both El-Minia and Suzan Mubarak University Hospitals and stress level. As evident in the table, less than two thirds $(63.3 \%$ and $63.9 \%$ ) of nurses with diploma degree and those who institute degree respectively and only more than half $(58.3 \%)$ of nurses who with baccalaureate degree had moderate stress.

More than one third (36.7\%) of nurses with diploma degree versus only one quarter $(25 \%)$ of those who institute degree and $41.7 \%$ of nurses who with baccalaureate degree had severe stress. These differences were highly statistically significant $(\mathrm{p}$ value $=0.005$ ).

Table (8) Displays the relation between the stress level of nurses in both El-Minia and Suzan Mubarak University Hospitals and their years of experience. As evident in the table, the nurses who had years of experience $>20$ years had the highest percentages $(71.4 \%)$ of moderate stress, compared to the other years of experience categories. More than one third $(36.5 \%)$ of nurses who had their years of experience less than 10 years had severe stress, compared to the other years of experience categories. These differences were not statistically significant ( $p$ value $=0.707$ ).

Table (9) Describes the relation between stress level of nurses in El-Minia and Suzan Mubarak University Hospitals and their shifts. As evident in the table, more than one third $(37.9 \%)$ of nurses at morning shift, about one third $(33.9 \%)$ of nurses at evening shift and only $30.0 \%$ of nurses at night shift had severe stress. There were no statistically significant differences between stress level at different shifts ( $p$ value $=0.062$ ). 


\section{Discussion:}

In the present study, more than two thirds of studied nurses who work in El-Minia University Hospital were females and all nurses who work in Suzan Mubarak University Hospital were females (table, 1). This result might be attributed to that majority of nurses who graduated from Secondary School of Nursing and clinical Institution was females. This is supported by (Walling, 2011) who mentioned that while the proportion of men entering the nursing profession has been growing, it remains a female-dominated occupation.

As regard frequency of stress causes, the most common causes of the severe stress from nurse's point of view were death and dying, work load, and uncertainty concerning treatment respectively (table, 2). This result is consistent with (Kelly, et al., 2007) who reported that stress resulting from extra work load, fear of injury and parents pursuing legal action. On the same line (McGrath, et al., 2003) reported that working with difficult patients, the nurses' feelings about death and dying, interpersonal conflicts, managing the patients' pain and the presence of the family also contribute to occupational stress.

The present study results show that severe stress in the general area was more than critical area at El-Minia and Suzan Mubarak Hospitals (table, 3). This might be due to shortage of nurses, increase in workload, increase number of patients, and shortage in equipments in the general area. This finding is consistent with (Strachota, et al., 2003) who reported that studies have established link between shortage of nurses, workload, and job stress. Also consistent with (Mohan\& Ashok, 2011) who stated that stress and depression are related to exhaustion and work load factors in various organization. Hart, (2003) said that heavy workload adversely affects nurses by threatening physical safety, lowering job satisfaction, and causing burnout.

In the present study results show that the highest percentages of nurses who working in ElMinia and Suzan Mubarak hospitals with severe stress were in age group less than 20 years, compared to the other age categories (table, 4). This might be attributed to the fact that the younger nurses have lower information about their duties and responsibilities, more times they exposed to stress, and nurses who are aged less than 20 years are in the period of role transition, and the transition of novice nurses into clinical practice is commonly perceived as stressful. This finding is consistent with (Kleinman, 2008) who mentioned that nurses at increased risk for role conflict tend to be those who are younger in age, have fewer years in their present staff position.

In addition, the result of the present study supported by (Keerthy, 2008) who mentioned that because some nurses are new in the field; receiving vague or insufficient information; not having much experience, they are not able to plan their job. Employees tend to be satisfied with their jobs when roles are clearly defined.

The present study results show that severe stress in female nurses was more than male nurses (table,5), from the researcher's point of view this could be attributed to multiple roles of female nurses so they unable to have clear information about their roles. These findings are consistent with (Mohan\& Ashok, 2011) who reported that all stress levels founds were high among the women employees. On the same line (Rathod et al., 2000, Al-Mishan, 2001) stated that females were more likely to report being stressed. Watson et al., (2008) stated that women scored higher than men on stress level.

As regard to marital status, the findings show that severe stress in married nurses was less than single nurses (table, 6 ). This may be attributed to the emotional stability in the married nurses. This finding is consistent with (Hastings, 2002) who concludes that there is some evidence of a relationship between negative emotions and stress. On the same line (Evandrou et al., 2002) stated that women assuming multiple roles results in work family conflict because time and energy are shared, clubbed and even extended across the two spheres of activity.

According to the present study findings, less than half of nurses that had severe stress with baccalaureate degree (table, 7). This may be attributed to the reality shock and anticipated job position. On the same line (Nemcek and James, 2007) concluded that individuals with higher educational levels have more responsibility and experience more stress.

According to the study findings, there is no significant relation between stress and years of experience, more than one third of nurses in the study sample with less than 10 years of experience had severe level of stress (table, 8). from the researcher's point of view this could be attributed to the experience do not determine number of times the nurses exposed to situational stressors and respond to stress depend on stress perception of each individual. This was consistent with (Mutiu, 2009) who study's findings did not show any significant relationship between job stress and length of work experience.

This was inconsistent with (Tummers et al., 2002) who stated that effects of stress depend on socio-demographic variables such as age and gender, 
on organizational variables such as length of service and position in the hierarchy at work, and on individual characteristics. On the same line (Abood, 1999) mentioned that some factors influencing the impact of stress were included (the duration of stressors, stress cumulative, individual perception, and cognitive appraisal).

In the current study, results show that the nurses who worked in the morning shift had the highest percentages of severe stress, compared to the other shifts (table, 9). This could be attributed to crowded work environment in units in the morning shift, workload in the morning shifts, and all routine procedures that is occur at the morning. This was consistent with (Natvig et al., 2007) who mentioned that increasing job demands was a problem for nurses. It could have consequences on patient safety in relation to inadequate time to properly test equipment and insufficient time for the preparation of medications. In addition, daily control routines in the morning could not be completed.

\section{Conclusions:}

Based on the results of the present study, it can be concluded that

- Less than two thirds of the studied sample has moderate level of the stress, and more than one third of the studied sample has severe level of the stress in El-Minia and Suzan Mubarak University Hospitals.

- There were statistically significant relation between level of education and stress level in the two hospitals. While there were no statistically significant differences between other demographic characteristics and stress levels in the two hospitals.

- The most common causes of stress with the nurses who worked at El-Minia and Suzan Mubarak University Hospitals are considered death, dying, work load, and uncertainty concerning treatment respectively.

\section{Recommendations:}

Based on results of the present study it can be recommended that:

1. Director of nursing should plan an orientation program to orient nurses about hospital policy, rules, regulations, job description and responsibilities, other's expectation, facilities and equipment and performance evaluation standard, to know their roles and reduce their stress.

2. Organizing stress management program that focuses on different categories of nurses at all hierarchical levels.
3. The shortage of the staff nurse should be managed by the nurse manager to meet the needs of the patients and reduce workloads that decrease nurse's work stress.

\section{References:}

1. Abood. S. A, (1999): Levels and sources f stress in Baccalaureate Nursing students at higher Institute of Nursing in Assiut University, pp.1112.

2. Al-Mishan. A. S, (2001): "Source of stress on the job among Kuwaiti and non-Kuwaiti government employees", Journal of King Saud University: Admin Sciences 2001; 13 (1), pp. 67-11

3. Alves. S. L, (2005): A study of occupational stress, scope of practice, and collaboration in nurse anesthetists practicing in anesthesia care team settings. AANA Journal, 73(6), pp. 443452.

4. Cassem, N. H, \& Hackett, T. P, (1981): Sources of tension for the CCU nurse. American Journal of Nursing, 1972, 72, 1426-1430.

5. Chou. S, Boldy. D \& Lee. A, (2003): Factors influencing residents' satisfaction in residential aged care. Gerontologist 43 (4), pp.459-472.

6. Devente. W, Kamphuis. J \& Emmelkamp. P, (2006): Alexithymia, Risk factor or consequence of work-related stress? Psychotherapy and Psychosomatics, 75(5), 304-11. Retrieved September 8, 2007, from ProQuest Psychology Journals database. (Document ID: 1135696381).

7. Evandrou M, Glaser. K \& Henz. U (2002): Multiple Role Occupancy in Midlife: Balancing Work and Family Life in Britain, the Gerontologist. Volume 42, issue 6, pp. 78-189.

8. Hannan. S, Norman. I. J\& Redfern. S, (2001): Care work and quality of care for older adults. Reviews in Clinical Gerontology, pp. 11, 189203

9. Hart. P. D, (2003): Patient-to-nurse staffing ratios: Perspectives from hospital nurses. A research study conducted on behalf of AFT Healthcare. Retrieved May 5, 2006, available @ http://www.aft.org./healthcare/ pubsreports/index.htm.

10. Hastings. R. P, (2002): Do challenging behaviors affect staff psychological well-being? Issues of causality and mechanism. American Journal on Mental Retardation, 107(6), pp.455467.

11. Keerthy. N, (2008): Assessment the difference between high and low criterion groups of emotional intelligence with regard to 
occupational stress available @ http://thebigbees.com/discussions.aspx

12. Kelly. A, Carey. S, McCarthy. S \& Coyle. C, (2007): Challenging behaviour: Principals' experience of stress and perception of the effects of challenging behaviour on staff in special schools in Ireland. European Journal of Special Needs Education, 22(2), pp. 161-181.

13. Kleinman. C, (2008): Role Conflict and the Nurse, Part I, available @ http://www.w3c.org/TR/1999/REC-html401 19991224/loose.dtd

14. Laschinger, H. K. S., \& Leiter, M. P. (2006). The impact of nursing work environments on patient safety outcomes: The mediating role of burnout/engagement. JON A the Journal of Nursing Administration, 36(5), 259-267.

15. Leiter. M \& Maslach. C, (2005): Six strategies for improving your relationships with work. San Francisco, CA: Jossey-Bass Publishers. Summer, 24(2), pp. 48-51.

16. McCauley, K., \& Irwin, R. S. (2006). Changing the work environment in intensive care units to acheive patient focused care: the time has come. American Journal of Critical Care, 15(6), 541548

17. McGrath. A, Reid. N \& Boore. J, (2003): Occupational stress in nursing. International Journal of Nursing Studies, 40(5), pp. 555.

18. Mohamed. F, Yousr. A \& Wafaa. M, (2011): Pediatric Nurses' Stresses in Intensive Care Units and Its Related Factors, Journal of American Science 2011; 7(9), pp. 304-315.

19. Mohan. N\& Dr.J.Ashok, (2011): Global Journal of Management and Business Research, pp. 1-5.

20. Mutiu. S,(2009): The Relationship between Job Stress and Life Satisfaction among NonAcademic University Staffs, , the 8th International Postgraduate Research Colloquium: Interdisciplinary Approach for Enhancing Quality of Life IPRC Proceedings, Department of Psychology International Islamic University aMalaysi available@bsris.swu.ac.th/iprc/8th/013_22_5_M utiu.pdf

21. Natvig. G, Gundersen. D \& Berland. A (2007): Patient safety and job-related stress: A focus group study, Department of Nursing Education, Stord/Haugesund University College, Bjørnsonsgate 45, Haugesund 5528, Norway Haugesund Hospital, Helse Fonna, Norway, journal of Intensive and Critical Care Nursing, volume 24, issue 2, April, pp. 90-97

22. Nemcek. M. A \&James. G. D, (2007): Relationships among the nurse work environment, self-nurturance and life satisfaction. J. Adv. Nurs. 59(3), pp.240-247.

23. Rathod. S, Roy, Leena, Ramsay. M, Das, M, Birtwistle. J \& Kingdom. D, (2000): “A survey of stress in psychiatrists working in the Wessex Region", Psychiatric Bulletin, pp. 24: 133-136.

24. Strachota. E, Normandin. P. O,'Brien. N, Clary. M \& Krukow, B. (2003): Reasons registered nurses leave or change employment status. Journal of Nursing Administration, 33(2), pp. 111- 117.

25. Sveinsdottir. H, Biering. A \& Ramel. A, (2006): "Occupational stress. job satisfaction. and working environment Icelandic nurses: A cross-sectional questionnaire survey". International Journal of Nursing Studies. 43(7), pp. 875-889.

26. Tummers. G, Landeweerd. J \& Merode. G, (2002): Work organization, work characteristics and their psychological effects on nurses in the Netherlands. International Journal of Stress Management, pp.9, 183-206.

27. Tyson. P. D \& Pongruengphant. R, (2004): Five-year follow-up study of stress among nurses in public and private hospitals in Thailand. International Journal of Nursing Studies, 41(3), pp. 247-254.

28. Walling. P, (2011): How did Nursing Become a Female Profession available @ http://www.themale-nurse.com/2011/06/how-did-nursingbecome-female.html

29. Watson. R, Eric .Gardiner, Richard .Hogston, Helen. Gibson, Anne .Stimpson, Robert. Wrate \& Ian Deary, (2008): A longitudinal study of stress and psychological distress in nurses and nursing students: Blackwell Publishing Ltd, Journal of Clinical Nursing, pp. 18, 270-278. 